\title{
A new ranking scheme for the institutional scientific performance
}

\author{
Selçuk Bilir*, Ersin Göğüş ${ }^{1}$, Özgecan Önal Taş², Talar Yontan² \\ Department of Astronomy and Space Sciences, Faculty of Sciences, Istanbul University, 34119, ${ }^{1}$ Faculty of Engineering and Natural Sciences, \\ Sabancı University, 34956, Orhanlı-Tuzla, ${ }^{2}$ Department of Astronomy and Space Sciences, Istanbul University, Graduate School of Science \\ and Engineering, 34116, Beyazit, Istanbul, Turkey
}

\begin{abstract}
We propose a new performance indicator to evaluate the productivity of research institutions by their disseminated scientific papers. The new quality measure includes two principle components: The normalized impact factor (IF) of the journal in which paper was published, and the number of citations received per year since it was published. In both components, the scientific impacts are weighted by the contribution of authors from the evaluated institution. As a whole, our new metric, namely, the Institutional Performance Score (IPS) takes into account both journal based impact and articles specific impacts. We apply this new scheme to evaluate research output performance of Turkish institutions specialized in astronomy and astrophysics in the period of 1998-2012. We discuss the implications of the new metric, and emphasize the benefits of it along with a comparison to other proposed institutional performance indicators. This study contains a new bibliometric indicator were developed to measure the scientific research performance of the institutions.
\end{abstract}

Keywords: Bibliometrics, impact, research evaluation, statistical analysis

\section{INTRODUCTION}

A natural extension of evaluating the research performance of individual scientists is to evaluate the research output productivity of research institutes. This is, however, a more challenging task than assessing the output records of an individual scientist for various reasons. First of all, the number of scientists affiliated varies remarkably among institutes. This is easily handled in the evaluation of the research performance of an institute by normalizing the research outputs with the number of affiliated researchers. Another important factor is the impact of the research output. At this front, the h-index, ${ }^{[1]}$ and some of its variants ${ }^{[2-8]}$ are usually employed.

*Address for correspondence:

E-mail: sbilir@istanbul.edu.tr

\begin{tabular}{|l|l|}
\hline \multicolumn{2}{|c|}{ Access this article online } \\
\hline Quick Response Code: & Website: \\
\hline & www.jscires.org \\
& DOl: \\
& $10.4103 / 2320-0057.167254$ \\
\hline
\end{tabular}

Along with the wider use of advanced technology and methodologies in scientific research, the nature of research teams is also evolving. Unlike a few decades ago, scientific investigations performed by teams of about 10 scientists or more are not uncommon. The size of research teams in some cases can be as large as hundreds, such as, the Large Hadron Collider collaboration at $\mathrm{CERN}^{1}$, which includes scientists affiliated with many different institutions. In the dissemination of these scientific efforts (most commonly in the form of research articles), the contribution of each team member (i.e., co-author) is not usually reported explicitly. Therefore, it would not be a fair evaluation of the respective institutions when these large collaboration articles are assessed without author contributions are taken into considerations. To account for authorship credit, various ways were proposed, such as, the harmonic author credit ${ }^{[9]}$ and the $i^{\text {th }}$ author credit, ${ }^{[10]}$ both of which credits the author based on the rank in the author list, or the fractional author credit ${ }^{[10]}$ which credits all authors equally.

There have been numerous extensive studies for the scientific productivity evaluations of research institutions.

${ }^{1}$ http://lhcb-public.web.cern.ch/lhcb-public/ 
Vieira and Gomes ${ }^{[1]}$ investigated research impact for scientific institutions using an indicator that includes the paper productivity as well as their citation performance. Batista et al. ${ }^{[12]}$ proposed a measure that is interrelated to the h-index: They introduced $h_{I}$ which is the ratio of the square of h-index of the institutional papers to the number of authors of these articles. Abramo et al. ${ }^{[13]}$ derived an indicator which is obtained by normalizing the institutional h-index with the number of full-time research personnel of the institute. Franceschini and Maisano ${ }^{[14]}$ suggested a structured technique to evaluate the scientific output of research groups, in which they employ h-index as the key ingredient. Recently, Franceschini et al. ${ }^{[15]}$ proposed the success index for evaluating research institutions which primarily takes into account institutional papers with greater citation records. Boell and Wilson ${ }^{[16]}$ proposed a ranking scheme based on the square of the journal impact factors (IFs). Levitt and Thelwall ${ }^{[17]}$ introduced a metric that incorporates weighted sum of the article citations and journal IF, somehow similar to our proposed indicator. However, their metric involves an arbitrary weighting constant that it applied to both cumulative citation counts and journal IFs. Moreover, their metric does not involve the author contribution factor. It is, therefore, important to note the fact that none of these performance indicators involves the effects of all the above-mentioned factors completely, in particular, the self-impact of an article and author contribution.

Here, we propose a new evaluation scheme for the institutional research productivity that takes into account the scientific impact of the output as well as the extent of the scientific contribution of its researchers. We introduce this new Institutional Performance Score (IPS) scheme in the next section. Then, we apply our proposed performance indicator method to the research outputs of institutions performing research in astronomy and astrophysics in Turkey, based on their article productivity between 1998 and 2012. Finally, we discuss the implications of our results and compare the results obtained with this new scheme and through other techniques in the last section.

\section{SUBJECTS AND METHODS}

\section{The Definition of the Institutional Performance Score}

As we have outlined in the first section, there is currently no quality indicator to rank scientific productivity of research institutions that takes into account scientific impact and author contribution at the same time. We introduce below our article productivity based new ranking scheme, which we call the IPS, which consists of two additive terms: (i) IF of journal for the year that the article has been published multiplied with the contribution of each co-author (AC) to the institutional article, and (ii) the ratio of the number of citations received ( $\left.n_{\text {citations }}\right)$ to the number of years passed since the paper has been published $\left(n_{\text {years }}\right)$, and also multiplied by AC.

$\mathrm{IPS}=\frac{1}{N} \sum_{i=1}^{\mathrm{N}}\left((\mathrm{IF})_{i}+\frac{n_{\text {citations }, \mathrm{i}}}{n_{\text {years, } \mathrm{i}}}\right) \times(\mathrm{AC})_{i}$

Where, $N$ is the total number of institutional articles published. In this scheme, the AC parameter is simply the ratio of the number of co-authors from a particular institute to the total number of co-authors. For example, if an article is published by five researchers; three of them are from Institute $A$ and two of them from Institute B, then the author contribution of this paper to Institute $A$ is $3 / 5$ and that to Institute $B$ is $2 / 5$. The latter term represents the scientific impact of an article, which diminishes over time if it is not cited at a steady pace. Effectively, this indicator combines author contribution added impact gained by the journal in which a particular article was published, and by the article itself.

\section{The Data}

In order to obtain the complete dataset for astronomy and astrophysics research papers, we used Thomson Reuters Web of Knowledge ${ }^{2}$, which includes 12 different databases of single and interdisciplinary citation indices. This database contains the list of all journals covered in Science Citation Index (SCI) and provides the citation counts without self-citations for individual papers since 1980 to present day. In the database, we have identified 1702 publications in "Astronomy and Astrophysics" whose authors or co-authors were based in Turkey and published in 56 SCI journals in the period from 1980 to 2012. After excluding papers with overlapping fields, such as physics particles fields, geosciences multidisciplinary, meteorology atmospheric sciences, engineering aerospace, geochemistry geophysics, mathematics interdisciplinary applications, and remote sensing, the total number of publications was reduced to 1062 . According to document types, these 1062 papers were divided into seven groups: Articles (976), proceedings (37), letters (16), reviews (15), errata (10), research notes (7), and editorial notes (1). It was also found that 37 of these studies were presented at meetings before they were published, 10 of them were

${ }^{2}$ http://apps.webofknowledge.com 
corrected then re-published. Due to these reasons, we only considered the articles, letters, reviews, and editorial notes, which resulted in a sample size of 1015 publications. In Figure 1, we present the distribution of these 1015 publications over time. Note that 782 of these papers had the leading author from Turkish institutions, while in 233 papers; the leading authors were from international institutions.

It can be seen from Figure 1 that there is a prominent increment in a number of publications that were published in SCI journals starting from about 2000. Bilir et al. ${ }^{[18]}$ suggested that this increase in scientific productivity was motivated by the application of the improved academic assignment criteria in 1998, common use of internet, and larger scale research opportunities provided by scientific research units of the universities. Therefore, in our study here we analyzed research papers that published between the years of 1998 and the end of 2012. We should note the important fact that the number of citations for each publication was determined as of 31 August 2013. This way, even the latest publications had about 1-year of visibility, since papers are usually published in SCI journals can take a couple of months before they are listed on Web of Knowledge.

Astronomy and astrophysics studies in Turkey are currently conducted in three main Departments such as Astronomy and Space Sciences in Ankara, Ege, Erciyes, and Istanbul Universities; Astronomy and Space Technologies in Akdeniz, Çanakkale Onsekiz Mart Universities (COMU), and Physics in Boğaziçi (BOUN); Çukurova, Istanbul Kültür, Middle East Technical (METU), and Sabanc1 Universities.

Note that astrophysical research has also been conducted at various subdivisions of the Turkish Scientific and Technological Research Council (TÜBİTAK) 3 . We have identified 749 papers that researchers based in Turkey have been leading authors or co-authors and have been published in SCI journals between 1998 and 2012. Researchers from 48 institutions contributed to these 749 publications. When we consider the papers with leading authors based in Turkey, the number of papers published within the same time frame reduces to 564 , which were contributed by researchers from 37 institutions inside Turkey.

\section{RESULTS}

We apply our proposed institutional performance indicator to the Turkish institutions performing research

\footnotetext{
${ }^{3}$ www.tubitak.gov.tr
}

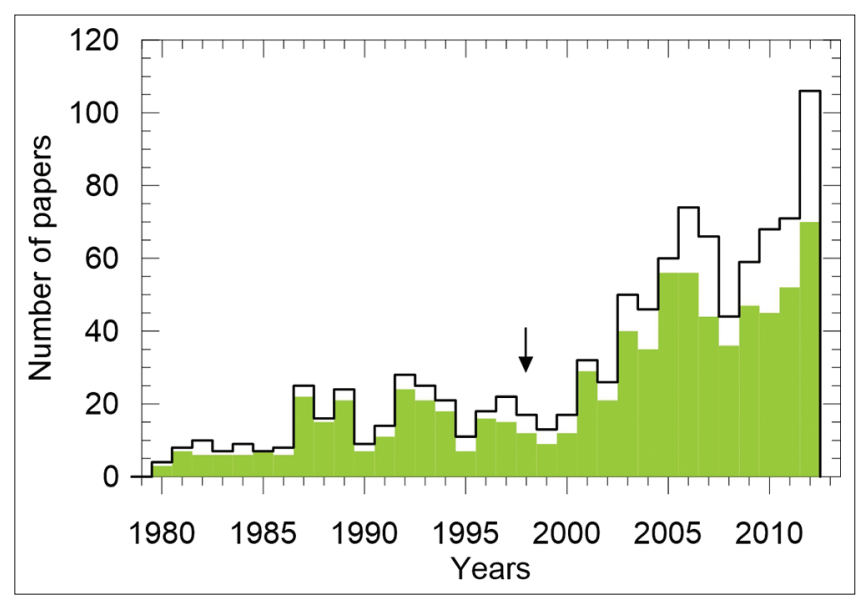

Figure 1: Distribution of research papers in astronomy and astrophysics which were published in Science Citation Index journals from 1980 to 2012. Green histograms represent the same distribution for the papers with the leading authors from institutions in Turkey

in astronomy and astrophysics, and disseminate their outputs in the form of scientific articles. Note that the IF of the journal in the year that a paper is published is one of the essential inputs for our new performance indicator definition. For this purpose, the IFs for the nine mostly preferred SCI journals between 1998 and 2012 were compiled and presented in Table 1. In the bottom row of Table 1, we provide the 15-year averages of annual IFs for each of these nine journals.

\section{Application of the Institutional Performance Score}

We present in Table 2, the resulting IPS of nine leading Turkish institutions, along with their number of publications, each component of the IPS, namely IF $\times$ AC (represented with (1) in Table 2$)$ and $\left(n_{\text {citations }} / n_{\text {years }}\right)$ $\times$ AC (2) in Table 2), as well as their institutional h- and the other indices. The table is formed in such a way that the upper part is for all 749 publications, and the lower part is formed by considering 564 publications whose leading author reside in Turkey. The institutes in both portions of Table 2 are ranked according to their IPS values.

We find that Sabanc1 University appears on top of the list in both publication categories, followed by Ege, which produced the largest number of institutional publications in our sample. It is noteworthy that Ege University was founded in 1962 while Sabanc1 University in 1999 and the average number of researchers in Ege have been much larger than that in Sabanc1 University. Our proposed performance indicator is not biased by such contrasts since we normalize the total quantities by the number of papers 
Table 1: Impact factors of SCI journals in astronomy and astrophysics between 1998 and 2012

\begin{tabular}{lccccccrrr}
\hline Years & MNRAS & A and A & ApJ & NewA & IJMPD & AN & Ap and SS & PASA & AJ \\
\hline 1998 & 3.960 & 1.630 & 1.953 & 2.912 & 0.732 & 0.518 & 0.234 & 0.419 & 2.003 \\
1999 & 4.548 & 2.252 & 2.543 & 2.947 & 1.064 & 0.600 & 0.275 & 0.868 & 2.876 \\
2000 & 4.685 & 2.790 & 2.822 & 2.241 & 1.051 & 0.410 & 1.189 & 1.028 & 3.604 \\
2001 & 4.681 & 2.281 & 5.921 & 2.348 & 1.242 & 0.553 & 0.274 & 0.951 & 3.018 \\
2002 & 4.671 & 3.781 & 6.187 & 3.108 & 1.507 & 0.786 & 0.383 & 0.898 & 5.119 \\
2003 & 4.993 & 3.843 & 6.604 & 3.866 & 1.618 & 1.199 & 0.522 & 1.057 \\
2004 & 5.238 & 3.694 & 6.237 & 2.171 & 1.500 & 0.906 & 0.597 & 1.158 \\
2005 & 5.352 & 4.223 & 6.308 & 1.921 & 1.225 & 0.871 & 0.495 & 1.735 \\
2006 & 5.057 & 3.971 & 6.119 & 2.220 & 1.651 & 1.399 & 0.771 & 1.588 \\
2007 & 5.249 & 4.259 & 6.405 & 1.714 & 1.870 & 1.461 & 0.834 & 1.390 \\
2008 & 5.185 & 4.153 & 6.331 & 1.784 & 1.741 & 1.261 & 1.283 & 2.564 \\
2009 & 5.103 & 4.179 & 7.364 & 1.675 & 1.046 & 1.186 & 1.404 & 3.786 \\
2010 & 4.888 & 4.425 & 6.063 & 1.632 & 1.109 & 0.842 & 1.437 & 4.769 \\
2011 & 4.900 & 4.587 & 6.024 & 1.411 & 1.183 & 1.012 & 1.686 & 2.490 \\
2012 & 5.521 & 5.084 & 6.733 & 1.850 & 1.030 & 1.399 & 2.064 & 4.555 \\
Average & 4.935 & 3.677 & 5.574 & 2.253 & 1.305 & 0.960 & 0.897 & 6.024 \\
\hline
\end{tabular}

MNRAS = Monthly Notices of the Royal Astronomical Society, A and A=Astronomy and Astrophysics, ApJ=Astrophysical Journal, NewA=New Astronomy, IJMPD = International Journal of Modern Physics D, AN=Astronomische Nachrichten, Ap and SS=Astrophysics and Space Science, PASA $=$ Publications of the Astronomical Society of Australia, AJ=Astronomical Journal

Table 2: The list of nine leading institutions with their number of papers $(n)$, total author contribution corrected journal impact (1)), author contribution corrected individual impact (2)), the ratio of (2) to (1), the IPS for all publications (upper portion) and for the publications with leading authors based in Turkey (lower portion). We also list few other performance indicators, namely h-index, g-index, AR-index, and IF $^{2}$

\begin{tabular}{|c|c|c|c|c|c|c|c|c|c|c|}
\hline Rank & Institution & $N$ & (1) & (2) & (2) /1 & IPS & h-index & g-index & AR-index & $\mathrm{IF}^{2}$ \\
\hline 1 & Sabancı & 105 & 216 & 115 & 0.53 & 3.15 & 24 & 35 & 12.45 & 40 \\
\hline 2 & Ege & 135 & 305 & 104 & 0.34 & 3.03 & 15 & 23 & 7.63 & 27 \\
\hline 3 & BOUN & 62 & 106 & 32 & 0.30 & 2.23 & 12 & 18 & 5.18 & 17 \\
\hline 4 & COMU & 115 & 180 & 72 & 0.40 & 2.19 & 18 & 25 & 8.74 & 26 \\
\hline 5 & İstanbul & 122 & 170 & 88 & 0.52 & 2.11 & 20 & 28 & 8.86 & 27 \\
\hline 6 & METU & 120 & 191 & 48 & 0.25 & 1.99 & 20 & 33 & 11.60 & 28 \\
\hline 7 & TÜBITAK & 62 & 80 & 15 & 0.19 & 1.53 & 12 & 18 & 7.03 & 21 \\
\hline 8 & Ankara & 71 & 75 & 31 & 0.41 & 1.49 & 11 & 23 & 8.64 & 15 \\
\hline 9 & Akdeniz & 45 & 45 & 16 & 0.36 & 1.36 & 11 & 15 & 5.15 & 17 \\
\hline Rank & Institution & $N$ & (1) & (2) & (2) /1 & IPS & h-index & g-index & AR-index & $\mathrm{IF}^{2}$ \\
\hline 1 & Sabancı & 43 & 140 & 76 & 0.54 & 5.02 & 14 & 23 & 8.21 & $\overline{31}$ \\
\hline 2 & Ege & 93 & 255 & 85 & 0.33 & 3.66 & 11 & 18 & 6.07 & 25 \\
\hline 3 & İstanbul & 62 & 115 & 68 & 0.59 & 2.95 & 14 & 19 & 6.54 & 20 \\
\hline 4 & METU & 66 & 151 & 32 & 0.21 & 2.77 & 13 & 21 & 5.98 & 24 \\
\hline 5 & COMU & 82 & 159 & 59 & 0.37 & 2.66 & 11 & 16 & 5.49 & 19 \\
\hline 6 & BOUN & 41 & 83 & 25 & 0.30 & 2.63 & 9 & 15 & 4.62 & 11 \\
\hline 7 & TÜBITAK & 31 & 63 & 11 & 0.17 & 2.39 & 7 & 11 & 3.10 & 15 \\
\hline 8 & Akdeniz & 24 & 33 & 13 & 0.39 & 1.92 & 6 & 8 & 3.77 & 9 \\
\hline 9 & Ankara & 40 & 52 & 15 & 0.29 & 1.68 & 6 & 10 & 2.80 & 8 \\
\hline
\end{tabular}

IPS = Institutional Performance Score, IF=Impact factor

published. With respect to the individual impact (2) in Table 2), Sabanc1 again earns the first rank, followed by Ege and Istanbul. Another important ranking tool here is the ratio of (2) to (1), that is, the fraction of the collective impact of scientific papers within the collective impact they gained by their respective journals. In this scheme, Sabanc1 leads, and closely followed by Istanbul University.
Sabanc1 University ranks on top also in other performance indicators. It is striking to note in Table 2 that METU, Istanbul and COMU the second, third and fourth places, respectively, in their respective $\mathrm{h}$-index, ${ }^{[1]} \mathrm{g}$-index, ${ }^{[3]}$ AR-index, ${ }^{[5]}$ and $\mathrm{IF}^{2[16]}$ rankings. In the IPS ranking, METU, Istanbul, and COMU rank sixth, fifth and fourth, respectively. 
We present in Figure 2, the average author contribution (AC) for each institution in both publication groups. We find that the average author contribution ratios from Turkish institutions to all publications vary between 0.34 and 0.70 . For all 749 publications, Turkey resident author contribution to research papers mostly comes from BOUN, Ege, and COMU [Figure 2a]. There are four institutions that were found to pass the author contribution ratios of 0.50 , while Sabanc1 University remains below this proportion, even though with the highest citation value received for research papers. The author contribution ratios for publications with domestic leading authors varied between 0.50 and 0.87. In this category, BOUN and Ege earn the first place, followed by COMU with 0.84 and Ankara University with 0.74 [Figure 2b]. Also in Figure 2, we present the number of citations per papers and institutional h-indices of all nine Turkish institutions. When citations per papers were considered, Sabanc1 University leads in both publication categories with 16.36 and 14.53 citations per paper, respectively. It is followed by METU (11.74\%) and Istanbul (10.78\%) for

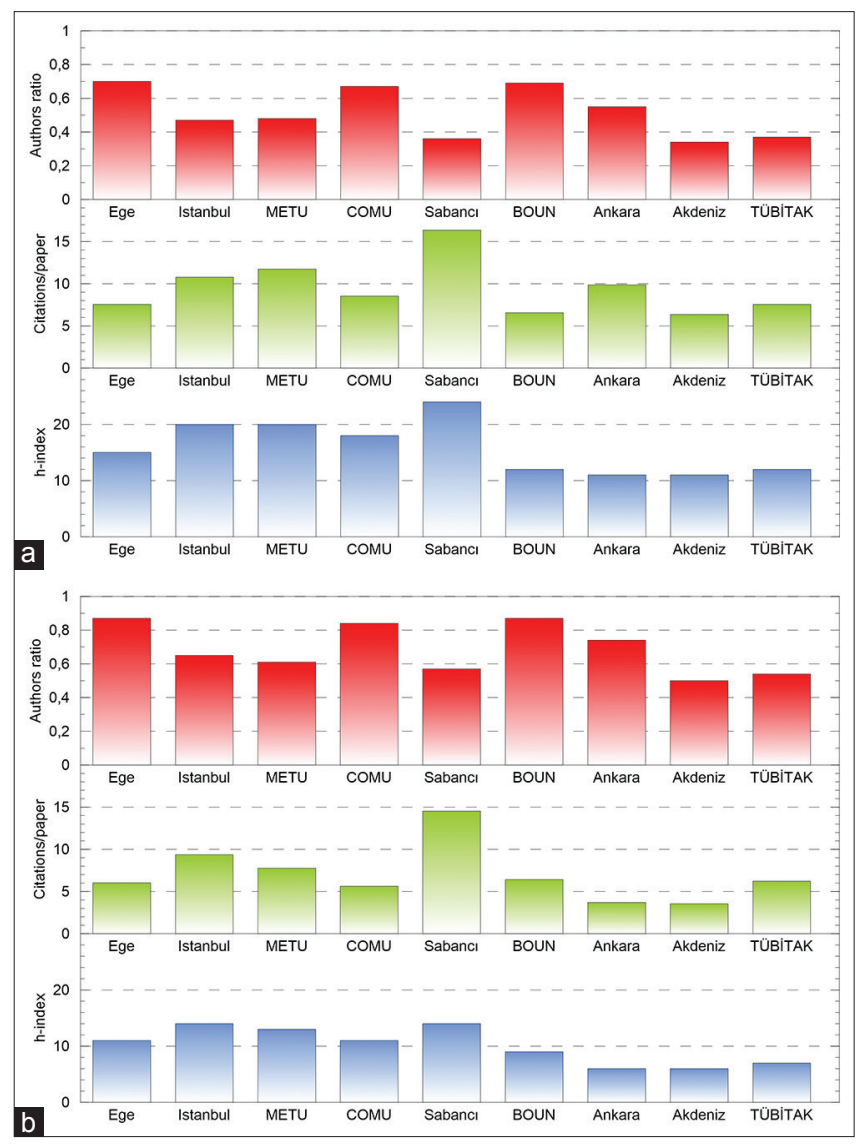

Figure 2: Average author contributions, citations per papers and h-index of institutions for all papers (a) and for papers with domestic leading authors (b) all publications and Istanbul (9.37\%) and METU (7.76\%) for publications with domestic leading authors.

Finally, we construct time evolution of IPS values of Turkish astronomy and astrophysics related research institutions with exceeding 100 publications. We also calculate h-index, g-index, AR-index, and $\mathrm{IF}^{2}$ for these five institutions to compare with our proposed performance indicator. As seen in Figure 3, the annual IPS of Sabanc1 University is mostly in the 4-8 bands over the course of our study from 1998 to 2012. Note the fact that Sabanc1 is a newly established institution, and astrophysical research started in 1999. Ege, METU, and Istanbul Universities lie around IPSs of 4. It is noteworthy that Ege exhibits a gradual increase trend until 2004. The IPS trends of other institutions appear between 2 and 4 . It is important to note that h-index, g-index, AR-index, and $\mathrm{IF}^{2}$ exhibit cumulative evolution in time while the IPS can evolve positive or negatively, depending on the scientific impact of research units.

\section{DISCUSSION}

We introduced a new quantitative indicator to evaluate the scientific performance of research institutions. Our

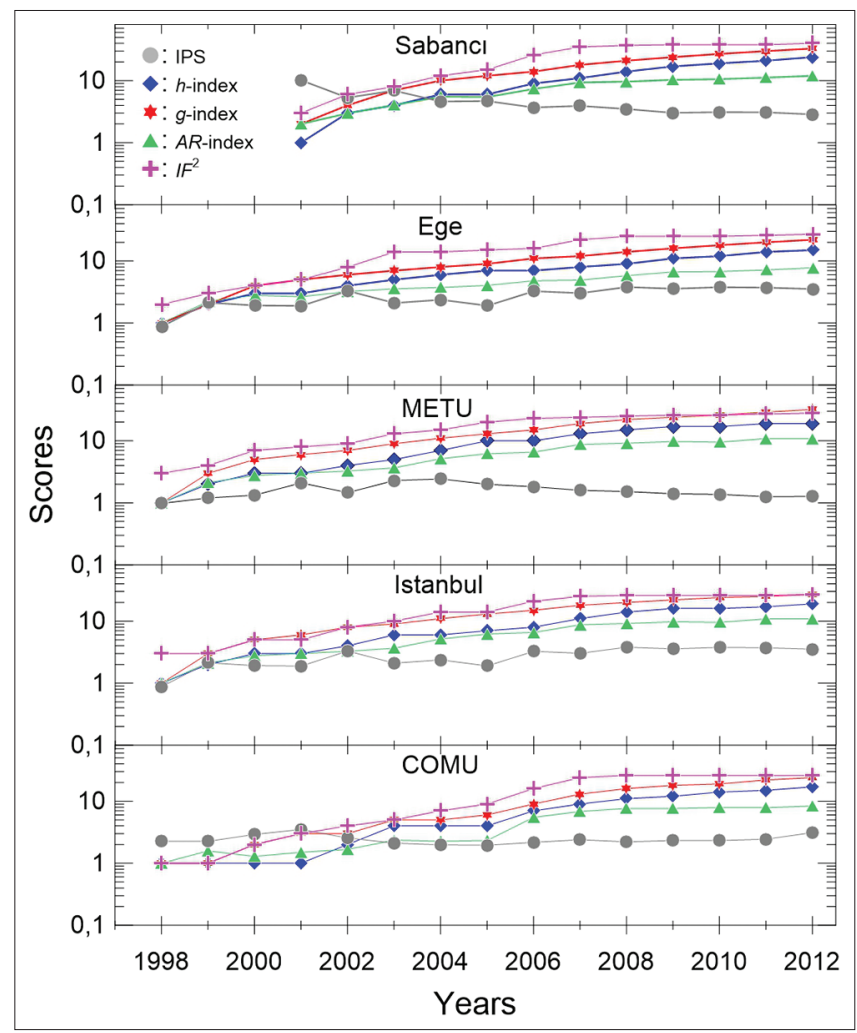

Figure 3: Evolution of Institutional Performance Score, h-index, g-index, AR-index, and $\mathrm{IF}^{2}$ for five institutions with more than 100 publications

J Scientometric Res. | May-Aug 2015 | Vol 4 | Issue 2 
proposed indicator consists of two crucial components: One is the author contribution weighted IF of the journal in which a paper has been published, and the other one is again author contribution corrected the number of citations received by the paper per each year since its appearance in the journal. In other words, the IPS value can be regarded as the institutional scientific impact of a research unit.

In the era of very high-speed communications and rather easy access to high-performance computation, scientists of today are greatly benefiting from the fact that geopolitical borders are no longer boundaries for scientific collaborations. As reaching out for international collaborations gets easier, the sizes of international research teams become eventually larger. When it comes to extensively large experimental efforts, such as, the Large Hadron Collider project at CERN, the size of collaborations can be as large as 1000 of researchers from 100's of different institutions. Therefore, it would not be trivial to assess the outcome of their collaborative effort (peer-reviewed papers) to a particular institution only. For this reason, we include the ratio of the number of co-authors from a particular institution to the total number of co-authors as a multiplicative weight for the IF of the journal in which a particular paper has been published.

The journal and its associated IF cannot provide a direct measure for the quality of a research topic. Some articles might end up in a journal with no page charge but has a low IF due to the lack of funding for publication costs. Nevertheless, there are, fortunately, numerous journals which require no publication charges but have high IFs, such as MNRAS in the field of astronomy and astrophysics. When folded with the ratio of contributing authors, the IF becomes a more sensitive quality indicator of a research paper.

Another important achievement indicator of a scientific paper is the number of citations received. It is unavoidable that a paper takes some time for its visibility before it is being referred by peer researchers. As years pass by, it will be eligible for further referral. In our parameterization, we consider the citation-based impact of a paper per the number of years passed so that the outcome is balanced for newly published papers, as well as those published a while ago and had already ample periods of time for their visibility.
We apply our new performance indicator scheme to the outputs of Turkish institution specialized in astronomy and astrophysics. We clearly find that commonly used h-index or its variants suggest slightly different rankings for the same sample since they involve primarily citations received by papers. This approach underestimates the performance of an institution which produced a modest number of highly cited papers. As we showed in Table 2, h-index, g-index, AR-index, and $\mathrm{IF}^{2}$ based ranking closely resemble each other. On the other hand, the IPS ranking is significantly different. Another important property of the use of IPS is that it can grow or decay, depending on the scientific performance of research institutes. Whereas, the other four indicators compared here evolve in time cumulatively.

We also investigated other proposed performance indicators. ${ }^{[11-13,15]}$ The indicators proposed by Abramo et al., ${ }^{[13]}$ requires the number of full-time equivalent staff of research institutions, which is, in most cases, not easy to obtain for the institutions other than the home institution of a researcher. The methods proposed by Batista et al. ${ }^{[12]}$ and Vieira and Gomes $^{[11]}$ differ from the pure h-index analysis, but still heavily based upon h-index parameters. Our proposed scheme, on the other hand, makes use of easily available input parameters, which can be extracted from various commonly used channels such as, Web of Knowledge.

\section{CONCLUSIONS}

We conclude that our proposed scientific impact indicator, IPS is a robust and distinguishing technique to evaluate research performance. Aside the final score, one can quantify the static impact (gained from journals) and dynamic impact, that is, the individual impacts of articles. While computing the IPS, one can also quantify the average author contribution of institutes that can be interpreted as a measure of openness/tendency for collaboration. Finally, our proposed performance indicator can be adopted to evaluate scientific impact of individual researchers. When summed over all publication of a researcher, this method would provide a sensitive comparison tool for performance evaluations.

\section{REFERENCES}

1. Hirsch JE. An index to quantify an individual's scientific research output. Proc Natl Acad Sci U S A 2005;102:16569-72.

2. Braun T, Glanzel W, Schubert A. A Hirsch-type index for journals. Scientometrics 2006;69:169-73.

3. Egghe L. Theory and practise of the g-index. Scientometrics 2006;69:131-52. 
4. Van Raan AF. Comparison of the Hirsch-index with standard bibliometric indicators and with peer judgment for 147 chemistry research groups. Scientometrics 2006;67:491-502.

5. Jin B, Liang L, Rousseau R, Egghe L. The R- and AR-indices: Complementing the h-index. Chin Sci Bull 2007;52:855-63.

6. Guan J, Gao X. Comparison and evaluation of Chinese research performance in the field of bioinformatics. Scientometrics 2008;75:357-79.

7. Vanclay J. Ranking forestry journals using the h-index. J Informetr 2008;2:326-34.

8. Schreiber M, Malesios CC, Psarakis S. Categorizing h-index variants. Res Eval 2011;20:397-409.

9. Hagen NT. Harmonic allocation of authorship credit: Source-level correction of bibliometric bias assures accurate publication and citation analysis. PLoS One 2008;3:e4021.

10. Liu $X Z$, Fang $H$. Fairly sharing the credit of multi-authored papers and its application in the modification of h-index and g-index. Scientometrics 2012;91:37-49.

11. Vieira ES, Gomes JA. A research impact indicator for institutions. J Informetr 2010;4:581-90.

12. Batista PD, Campiteli MG, Kinouchi O, Martinez AS. Is it possible to compare researchers with different scientific interests? Scientometrics 2006;68:179-89.
13. Abramo G, D'Angelo CA, Viel F. The suitability of $h$ and $g$ indexes for measuring the research performance of institutions. Scientometrics 2013;97:555-70.

14. Franceschini F, Maisano D. Analysis of the Hirsch index's operational properties. Eur J Oper Res 2010;203:494-504.

15. Franceschini F, Maisano D, Mastrogiacomo L. Evaluating research institutions: The potential of the success-index. Scientometrics 2013:96:85-101.

16. Boell SK, Wilson CS. Journal impact factors for evaluating scientific performance: Use of h-like indicators. Scientometrics 2010;82:613-26.

17. Levitt JM, Thelwall M. A combined bibliometric indicator to predict article impact. Inf Process Manag 2011;47:300-8.

18. Bilir S, Göğüş E, Önal Ö, Öztürkmen ND, Yontan T. Research performance of Turkish astronomers in the period of 1980-2010. Scientometrics 2013;97:477-89.

How to cite this article: Bilir S, Gögüs E, Tas ÖÖ, Yontan T. A new ranking scheme for the institutional scientific performance. J Sci Res 2015;4:70-6

Source of Support: Nil, Conflict of Interest: None declared 